\title{
Análisis financiero: una herramienta clave para una gestión financiera eficiente
}

\author{
Nava Rosillón, Marbelis Alejandra*
}

\section{Resumen}

El análisis financiero es fundamental para evaluar la situación y el desempeño económico y financiero real de una empresa, detectar dificultades y aplicar correctivos adecuados para solventarlas. El objetivo de este estudio es analizar la importancia del análisis financiero como herramienta clave para una gestión financiera eficiente. Es un estudio analítico con diseño documental basado en los fundamentos teóricos de Gitman (2003), Van Horne (2003), Elizondo y Altman (2003), entre otros. El análisis financiero se basa en el cálculo de indicadores financieros que expresan la liquidez, solvencia, eficiencia operativa, endeudamiento, rendimiento y rentabilidad de una empresa. Se considera que una empresa con liquidez es solvente pero no siempre una empresa solvente posee liquidez. El análisis financiero basado en cifras ajustadas por inflación proporciona información financiera válida, actual, veraz y precisa. Se concluye que el análisis financiero es una herramienta gerencial y analítica clave en toda actividad empresarial que determina las condiciones financieras en el presente, la gestión de los recursos financieros disponibles y contribuye a predecir el futuro de la empresa.

Palabras clave: Análisis financiero, empresa, gestión financiera, indicadores financieros.

\section{Financial Analysis: A Key Technique for Efficient Financial Management}

\begin{abstract}
Financial analysis is fundamental to assessing the real economic and financial situation and performance of a company, detecting difficulties and applying suitable correctives to solve them. The objective of this study is to analyze the significance of financial analysis as a key technique for efficient financial management. It is an analytical study with a documentary design based on the theories of Gitman (2003), Van Horne (2003), Brigham and Houston (2006), Elizondo and Altman (2003), among others. Financial analysis is based on estimating financial indicators that show the liquidity, solvency,

Recibido: 09-02-09. Aceptado: 20-10-09

* $\quad$ Economista. Magíster en Gerencia de Empresas, mención: Gerencia Financiera. Universidad del Zulia. Gerente Administrativo de la Empresa Agropecuaria "Santa Rita". Acreditada en el Programa de Promoción al Investigador. E-mail: marbejans@cantv.net; marbejans@hotmail.com.
\end{abstract}


operative efficiency, debt, yield and profitability of an enterprise. A business with liquidity is considered solvent, but a solvent business does not always have liquidity. Financial analysis based on figures adjusted for inflation provides valid, current, truthful and precise financial information. Conclusions are that financial analysis is a key management and analytic technique for all business activity that determines current financial conditions, the management of available financial resources and that it contributes to predicting the future of the enterprise.

Key words: Financial analysis, enterprise, financial management, financial indicators.

\section{Introducción}

Actualmente el proceso de transformación que trae consigo los avances tecnológicos, la automatización de los procesos, el desarrollo económico, el crecimiento de muchas empresas, dificulta la permanencia y el progreso de los negocios en su entorno. Las exigencias implícitas en estos cambios hacen indispensable que las unidades empresariales estén preparadas para gestionar sus recursos financieros de manera adecuada; de forma tal, que se tomen decisiones financieras racionales acordes con los objetivos de la empresa.

Sin embargo, las organizaciones son vulnerables a sufrir algún desequilibrio financiero imprevisto, caracterizado por insolvencia y poca liquidez, como producto de políticas financieras poco efectivas o por deficiencias en el desempeño estratégico, administrativo, productivo o financiero; por lo tanto, toda empresa debe conocer su condición económica y financiera para identificar los problemas existentes, variaciones importantes y los factores que los ocasionan, para ello debe disponer de herramientas apropiadas que le permitan detectar los errores y aplicar los correctivos adecuados, predecir el futuro y lograr una planeación más idónea.
El análisis o diagnóstico financiero constituye la herramienta más efectiva para evaluar el desempeño económico y financiero de una empresa a lo largo de un ejercicio específico y para comparar sus resultados con los de otras empresas del mismo ramo que estén bien gerenciadas y que presenten características similares; pues, sus fundamentos y objetivos se centran en la obtención de relaciones cuantitativas propias del proceso de toma de decisiones, mediante la aplicación de técnicas sobre datos aportados por la contabilidad que, a su vez, son transformados para ser analizados e interpretados.

La importancia del análisis financiero radica en que permite identificar los aspectos económicos y financieros que muestran las condiciones en que opera la empresa con respecto al nivel de liquidez, solvencia, endeudamiento, eficiencia, rendimiento y rentabilidad, facilitando la toma de decisiones gerenciales, económicas y financieras en la actividad empresarial.

El análisis financiero debe ser aplicado por todo tipo de empresa, sea pequeña o grande, e indistintamente de su actividad productiva. Empresas comerciales, petroleras, industriales, metalmecánicas, agropecuarias, turísticas, constructoras, entre otras, deben asumir el compromiso de llevarlo a cabo; puesto 
Análisis financiero: una herramienta clave para una gestión financiera eficiente Nava Rosillón, Marbelis Alejandra

que constituye una medida de eficiencia operativa que permite evaluar el rendimiento de una empresa.

Esta herramienta facilita el proceso de toma de decisiones de inversión, financiamiento, planes de acción, permite identificar los puntos fuertes y débiles de la organización así como realizar comparaciones con otros negocios, ya que aporta la información necesaria para conocer el comportamiento operativo de la empresa y su situación económica-financiera, para lo cual se fundamenta en los datos expuestos en los estados financieros, que son utilizados para calcular y examinar los indicadores financieros.

No obstante, el análisis financiero se debe realizar en forma sistemática de manera de determinar la liquidez y solvencia de la empresa, medir su actividad operativa, la eficiencia en la utilización de los activos, su capacidad de endeudamiento y de cancelación de las obligaciones contraídas, sus utilidades, las inversiones requeridas, su rendimiento y rentabilidad.

Sin embargo, el análisis financiero presenta algunas limitaciones inherentes a su aplicación e interpretación; puesto que se debe confirmar que la contabilización sea homogénea al realizar la comparación de cifras con empresas semejantes, debido a que las organizaciones muestran distinto nivel de diversificación en tamaño y tiempo de operatividad, en el nivel de internacionalización y en los criterios para la toma de decisiones contables, económicas y financieras. También, en lo respectivo a la interpretación de los indicadores financieros se pueden presentar dificultades para establecer criterios para su evaluación, ya que un resul- tado puede ser ambiguo en relación a la actividad productiva de la empresa.

Del mismo modo, se debe considerar el comportamiento de variables exógenas a la actividad empresarial como la inflación que afecta el valor actual de mercado de los activos y pasivos; algunos de ellos, generalmente, se omiten en el balance general o son valuados al costo depreciado de adquisición; por lo tanto, es necesario reexpresar los estados financieros antes de aplicar los indicadores financieros para lograr un análisis más completo y real de la situación de la empresa.

En base a lo expuesto anteriormente, se presenta este estudio que tiene como objetivo principal analizar la importancia del análisis financiero como herramienta clave para una gestión financiera eficiente; para ello es primordial describir el análisis financiero como fase gerencial; identificar los indicadores financieros pertinentes para efectuarlo, así como determinar las técnicas en las cuales se fundamenta, los efectos del fenómeno inflacionario en su aplicabilidad y analizar brevemente la importancia de las normas financieras internacionales en el uso de este útil instrumento gerencial.

Se trata de un estudio analítico con un diseño documental (Hurtado, 2007); que busca analizar la importancia y utilidad del análisis financiero como herramienta básica para llevar a cabo una gestión financiera eficiente, tomando como punto inicial los fundamentos teóricos de algunos autores como Gitman (2003), Van Horne (2003), Rubio (2007), Hernández, (2005), Brigham y Houston (2006), Pacheco et al (2002); Elizondo y Altman (2003); entre otros; los cuales presentan las nociones esenciales del análisis de 
los estados financieros de una empresa, sus objetivos, sus técnicas y los indicadores o razones financieras que permiten analizar e interpretar la información registrada en los estados financieros, mediante lo cual se logra detectar las fallas o deficiencias en el proceso gerencial.

\section{Análisis Financiero como Fase Gerencial}

Debido a los grandes y apresurados cambios en el entorno empresarial, los gerentes enfrentan la necesidad de poseer conocimientos de alto nivel que les permitan tomar decisiones rápidas y oportunas, ello requiere la aplicación de herramientas útiles para gestionar eficientemente sus empresas y alcanzar los objetivos establecidos.

Generalmente en las organizaciones se presentan problemas financieros que resultan difíciles de manejar; enfrentar los costos financieros, el riesgo, baja rentabilidad, conflictos para financiarse con recursos propios y permanentes, toma de decisiones de inversión poco efectivas, control de las operaciones, reparto de dividendos, entre otros.

Una empresa que enfrente un entorno difícil y convulsionado con los inconvenientes descritos anteriormente, debe implementar medidas que le permitan ser más competitiva y eficiente desde la perspectiva económica y financiera, de forma tal que haga mejor uso de sus recursos para obtener mayor productividad y mejores resultados con menores costos; razón que implica la necesidad de realizar un análisis exhaustivo de la situación económica y financiera de la actividad que lleva a cabo.
Para ello, es indispensable que los gerentes de las empresas conozcan los principales indicadores económicos y financieros y su respectiva interpretación, lo cual conlleva a profundizar y a aplicar el análisis financiero como base primordial para una toma de decisiones financieras efectiva. Esto sugiere la necesidad de disponer de fundamentos teóricos acerca de las principales técnicas y herramientas que se utilizan actualmente para alcanzar mayor calidad de la información financiera, mejorar el proceso de toma de decisiones y lograr una gestión financiera eficiente.

Hernández (2005), define el análisis financiero como una técnica de evaluación del comportamiento operativo de una empresa, que facilita el diagnóstico de la situación actual y la predicción de cualquier acontecimiento futuro; a su vez está orientado hacia la consecución de objetivos preestablecidos.

Este análisis se basa en la interpretación de los sucesos financieros ocurridos en el desarrollo de la actividad empresarial, para lo cual utiliza técnicas que una vez aplicadas llevan a una toma de decisiones acertadas; aunado a que contribuye a examinar la capacidad de endeudamiento e inversión de la empresa, tomando como punto de inicio la información aportada por los estados financieros.

El análisis financiero es un instrumento disponible para la gerencia, que sirve para predecir el efecto que pueden producir algunas decisiones estratégicas en el desempeño futuro de la empresa; decisiones como la venta de una dependencia, variaciones en las políticas de crédito, en las políticas de cobro o de inventario, así como también una expan- 
Análisis financiero: una herramienta clave para una gestión financiera eficiente Nava Rosillón, Marbelis Alejandra

sión de la empresa a otras zonas geográficas (Brigham y Houston, 2006).

En efecto, el análisis financiero es una herramienta clave para el manejo gerencial de toda organización, ya que contempla un conjunto de principios y procedimientos empleados en la transformación de la información contable, económica y financiera que, una vez procesada, resulta útil para una toma de decisiones de inversión, financiación, planeación y control con mayor facilidad y pertinencia, aunado a que permite comparar los resultados obtenidos por una empresa durante un lapso de tiempo determinado con los resultados de otros negocios similares.

Rubio (2007), enfoca el análisis financiero como un proceso que consiste en la aplicación de un conjunto de técnicas e instrumentos analíticos a los estados financieros, para generar una serie de medidas y relaciones que son significativas y útiles para la toma de decisiones; puesto que, la información registrada en los estados financieros por sí sola no resulta suficiente para realizar una planificación financiera pertinente o analizar e interpretar los resultados obtenidos para conocer la situación financiera de la empresa.

El análisis de los estados financieros se caracteriza por ser una operación fundamentada en la reclasificación, recopilación, obtención y comparación de datos contables, operativos y financieros de una organización, que mediante la utilización de técnicas y herramientas adecuadas busca evaluar la posición financiera, el desarrollo y los resultados de la actividad empresarial en el presente y pasado para obtener las mejores estimaciones para el futuro.
Con base a estas afirmaciones, se deduce que el análisis financiero es una fase gerencial que abarca varias etapas. Inicialmente, se centra en la utilización y conversión de la información contable registrada en los estados financieros; posteriormente, se emplea como herramienta para seleccionar la información más adecuada, prevenir situaciones contraproducentes y predecir el futuro, mediante el cálculo de indicadores y la aplicación de técnicas específicas; y por último, llega al diagnóstico y evaluación de las condiciones económicas y financieras en las cuales se encuentra operando la organización.

En este contexto, los objetivos del análisis financiero están encaminados a analizar las tendencias de las variables financieras involucradas en las operaciones de la empresa; evaluar su situación económica y financiera para determinar el nivel de cumplimiento de los objetivos preestablecidos; verificar la coherencia de la información contable con la realidad de la empresa; identificar los problemas existentes, aplicar los correctivos pertinentes y orientar a la gerencia hacia una planificación financiera eficiente y efectiva.

Pues, los objetivos del análisis financiero se fundamentan en la medición del nivel de solvencia, liquidez, rentabilidad y, en general, la situación financiera actual de la empresa; por ello, el cumplimiento cabal de estos objetivos está sujeto a la calidad de la información contable y financiera utilizada para su aplicación.

Para el desarrollo del análisis financiero se requiere del cálculo de indicadores o razones financieras, que permiten realizar un diagnostico de la situación económica y financiera del negocio. A jui- 
cio de Van Horne (2003), se utiliza una razón o índice que relaciona entre sí los elementos de información financiera reflejados en los estados financieros y de esta forma, se logra evaluar la condición y desempeño financiero de la empresa.

El método de cálculo de los indicadores financieros es el procedimiento de evaluación financiera más conocido y amplio; puesto que consiste en combinar o relacionar entre sí dos elementos que representan datos registrados en los estados financieros, con el fin de obtener un resultado que permite inferir acerca de aspectos que caracterizan dicha relación.

En virtud de esto, es importante ser muy cautelosos al momento de calcular los indicadores financieros, ya que los factores que afectan uno de los elementos que participan en la relación puede afectar al otro, lo cual puede provocar variaciones significativas en la realidad financiera del negocio. Esto se traduce en que se debe estar atento a cualquier cambio que pueda sufrir alguno de esos elementos o componentes, de manera de analizar efectivamente la variación que se refleja en el valor final del indicador.

De allí, que la importancia de calcular los indicadores financieros radica en la interpretación del valor que arroja cada indicador o razón, ya que ese valor por sí solo proporciona muy poca o ninguna información; y su cálculo no sólo se debe limitar a la aplicación de la fórmula sino que cada resultado numérico tiene un significado.

Así, los indicadores financieros suministran gran información acerca del funcionamiento y posición financiera de la empresa, básicamente cuando se calculan para una serie de períodos, esto permite determinar promedios y tendencias y también cuando son comparados entre varias empresas del mismo ramo; pues, sólo a través de los indicadores financieros es posible la comparabilidad de empresas de una misma actividad indistintamente de su tamaño.

Por otra parte, Pacheco et al. (2002); señalan que los indicadores financieros constituyen el resultado de las prioridades financieras tradicionales, relacionando los elementos del balance general y del estado de resultados; es así como permiten conocer la situación de la empresa en lo que respecta a liquidez, solvencia, eficiencia operativa, endeudamiento, rendimiento y rentabilidad.

Los resultados arrojados por el análisis financiero facilitan la posibilidad de analizar la evolución de la empresa en el tiempo, determinar la eficiencia en el uso de los recursos económicos y financieros y visualizar el desempeño de la gestión financiera de la empresa; puesto que ésta se relaciona fundamentalmente con la toma de decisiones referentes al tamaño y composición de los activos, al nivel y estructura de la financiación y a las políticas de dividendos establecidas en una empresa (Mallo y Merlo, 1995).

Básicamente, la gestión financiera engloba lo concerniente al dinero, a la inversión, administración y posesión del mismo, de manera que este sea manejado adecuadamente para que sea lucrativo; por ello su objetivo se centra en la maximización del valor de la inversión de los propietarios de la empresa.

En sus inicios el concepto de gestión financiera se limitaba a la administración de los fondos y sus funciones esta- 
Análisis financiero: una herramienta clave para una gestión financiera eficiente Nava Rosillón, Marbelis Alejandra

ban a cargo de una persona o del departamento de finanzas, pero con el transcurso del tiempo ha evolucionado mucho; tanto que en nuestros días se ha convertido en un concepto amplio y complejo.

Actualmente la gestión financiera incluye actividades significativas para alcanzar el éxito de una empresa; se encarga básicamente de la administración de los medios financieros, y para ello debe crear e implementar estrategias efectivas que le permitan obtener los recursos financieros, analizar los aspectos financieros que contienen las decisiones tomadas en otras áreas internas de la empresa, evaluar las inversiones requeridas para incrementar las ventas, analizar e interpretar la información financiera presentada en los estados financieros $y$ diagnosticar las condiciones económicas y financieras de la empresa.

Esto sugiere que el análisis financiero representa un instrumento fundamental en la labor del gerente financiero, ya que es el mecanismo para evaluar la situación de la organización en relación a liquidez, solvencia, eficiencia en las operaciones, nuevas estrategias de ventas, cobranzas, la necesidad de financiamiento y el rendimiento generado; así como también, permite verificar sí los objetivos financieros alcanzados están acordes con lo planificado.

Aunado a esto, el gerente financiero debe estar informado acerca de todos los acontecimientos financieros ocurridos día a día a nivel mundial, de manera que pueda determinar la posición financiera que ocupa su empresa con respecto al mercado en el cual se desenvuelve y estudiar como repercutirán esos hechos en su actividad empresarial.
En definitiva, la gestión en toda empresa se debe fundamentar en la utilización de las herramientas y técnicas más actualizadas que le permitan analizar su desempeño financiero en forma eficiente, con el propósito de tomar decisiones más acertadas, decisiones que sean efectivas basadas en información financiera útil, adecuada, oportuna y confiable que lleve al logro de los objetivos, a la permanencia de la empresa en el mercado y al éxito de su actividad productiva.

\section{Indicadores de la situación financiera de la empresa}

Actualmente, en la mayoría de las empresas los indicadores financieros se utilizan como herramienta indispensable para determinar su condición financiera; ya que a través de su cálculo e interpretación se logra ajustar el desempeño operativo de la organización permitiendo identificar aquellas áreas de mayor rendimiento y aquellas que requieren ser mejoradas.

Entre los indicadores financieros más destacados y utilizados frecuentemente para llevar a cabo el análisis financiero, se encuentran los siguientes: indicadores de liquidez y solvencia, indicadores de eficiencia o actividad, indicadores de endeudamiento y los indicadores de rentabilidad.

\subsection{Liquidez y solvencia}

Muchos autores hacen referencia a la liquidez, pocos a la solvencia, pero algunos refieren el concepto de liquidez con el término de solvencia (Rubio, 2007); razón por la cual es necesario dis- 
tinguir entre estas definiciones; pues, la liquidez implica mantener el efectivo necesario para cumplir o pagar los compromisos contraídos con anterioridad; mientras que la solvencia está enfocada en mantener bienes y recursos requeridos para resguardar las deudas adquiridas, aún cuando estos bienes no estén referidos a efectivo.

Para una empresa tener liquidez significa cumplir con los compromisos y tener solvencia refleja la disponibilidad que posee para pagar esos compromisos; esto indica que para que una empresa presente liquidez es necesario que sea solvente con anticipación.

Sin embargo, para Gitman (2003), la liquidez se mide por la capacidad que posee una empresa para pagar sus obligaciones a corto plazo en la medida que se vencen. Este autor considera que la liquidez está referida a la solvencia de la posición financiera general de la organización, lo que se traduce en la facilidad que tiene la empresa para pagar sus deudas.

No obstante, la liquidez es la capacidad inmediata de pago con la cual una empresa puede responder a sus acreedores; en tanto, la solvencia es la capacidad que tiene una empresa de responder en el corto plazo; cuya capacidad se refleja en la posesión de bienes que la empresa pueda disponer para cancelar los compromisos contraídos en corto tiempo.

Para que una empresa sea solvente debe estar dispuesta a liquidar los pasivos contraídos al vencimiento de los mismos; además, debe demostrar que está en capacidad de continuar con una trayectoria normal que le permita mantener un entorno financiero adecuado en el futuro.
De este modo, la solvencia se refleja en la tenencia de cantidades de bienes que una empresa dispone para saldar sus deudas, pero sí para esa empresa no es fácil convertir esos bienes en efectivo para realizar sus cancelaciones, entonces no existe liquidez; por ello, es importante destacar que una empresa con liquidez es solvente pero no siempre una empresa solvente posee liquidez.

Desde el punto de vista económico, la liquidez está dada por la facilidad o dificultad de convertir un activo en dinero efectivo en forma inmediata y sin que sufra pérdida significativa de su valor, esto indica que mientras más fácil sea convertir un activo en efectivo más líquido será ese activo.

Una empresa que requiera cancelar los compromisos contraídos puede obtener recursos financieros más rápidamente mediante la conversión de sus inventarios y cuentas por cobrar en efectivo; puesto que, una empresa se considera liquida en la medida que posee una mayor proporción de sus activos totales bajo la modalidad de activos circulantes; estos últimos, constituyen todo el dinero que se encuentra disponible en efectivo al momento de elaborar el balance general de la empresa, como el dinero en bancos y caja chica, las cuentas por cobrar en el corto plazo y las colocaciones próximas a su vencimiento.

En razón de ello, los niveles de liquidez de una empresa se pueden determinar mediante la aplicación del análisis financiero, el cual relacionando los elementos de información financiera aportados por los estados financieros permite calcular indicadores específicos que miden la liquidez y solvencia en una empre- 
Análisis financiero: una herramienta clave para una gestión financiera eficiente Nava Rosillón, Marbelis Alejandra

sa, como el capital de trabajo, la razón circulante y la razón prueba del ácido, que reflejan la capacidad de pago de la deuda circulante a corto plazo (Figura 1).

El capital de trabajo se refiere a la inversión que realiza una organización en activos circulantes o a corto plazo: efectivo, valores realizables, inventario (Brigham y Houston, 2006). Esto se traduce en que el capital de trabajo incluye todos los recursos que destina una empresa diariamente para llevar a cabo su actividad productiva, referidos tanto a activos circulantes como a pasivos circulantes; cuya diferencia da lugar al denominado capital de trabajo neto.

Sin embargo, algunos autores consideran que el capital de trabajo más que un indicador es una medida de la liquidez general de la empresa (Gitman, 2003); ya que mientras mayor es el margen en que los activos circulantes cubren los compromisos a corto plazo mayor es la capacidad de pago generada por la empresa para la cancelación de las deudas a su vencimiento; esto se fundamenta en que los activos circulantes constituyen entradas de efectivo y los pasivos circulantes, desem- bolsos de efectivo; entonces, resulta necesario disponer de capital de trabajo para garantizar el efectivo requerido para responder con el pago de las deudas contraídas al momento de su vencimiento.

La razón circulante permite determinar la capacidad de la empresa para cancelar sus deudas en el corto plazo relacionando los activos circulantes con los pasivos circulantes. El gerente financiero debe tener en cuenta que no siempre una razón circulante alta significa disponibilidad del efectivo requerido para las operaciones; ya que sí el inventario no puede ser vendido o las ventas a crédito no son cobradas a tiempo para obtener el efectivo requerido, entonces el alto valor expresado por la razón circulante puede ser incierto (Brigham y Houston, 2006).

Cabe considerar que una organización al presentar problemas financieros, comienza a implementar medidas como cancelar sus compromisos con mayor lentitud o solicitar préstamos a la banca, de esta manera el pasivo circulante se incrementa más rápidamente y se ubica por encima del activo circulante, esto hace que la razón circulante comience a

Figura 1

Indicadores de Liquidez

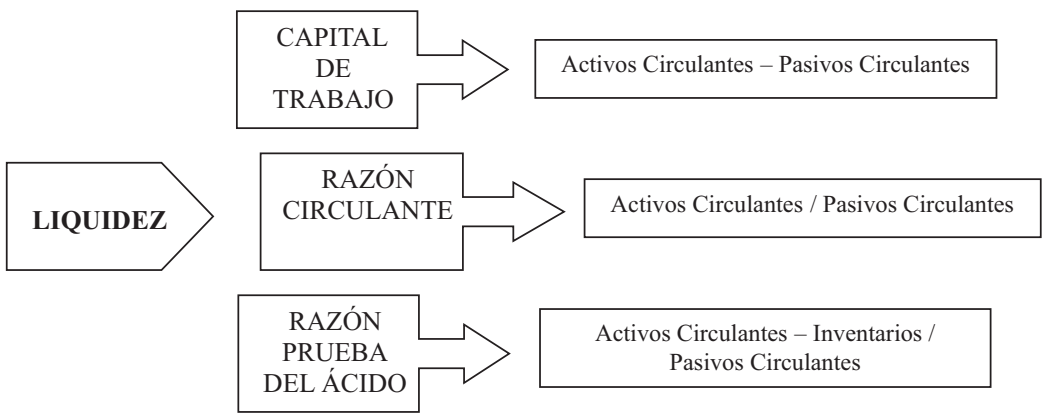

Fuente: Elaboración propia. 
disminuir, lo cual resulta desfavorable para cualquier actividad empresarial; pues, este es el indicador de liquidez y solvencia más confiable por expresar la tenencia de efectivo.

Por su parte, la razón prueba del ácido mide la suficiencia o no que posee la empresa para pagar en forma inmediata sus deudas en un momento dado; es similar a la razón circulante excepto que excluye el inventario, el cual es un activo menos líquido que el resto de los activos circulantes.

La importancia de determinar los indicadores de liquidez en una empresa radica en que proporcionan la información acerca del nivel de liquidez que posee la misma; pues se puede presentar una situación de iliquidez, en la cual la organización no dispone de efectivo para la cancelación de sus obligaciones, cuya situación es contraproducente a los objetivos de la gestión financiera.

La iliquidez acarrea consecuencias que implican limitaciones en la capacidad de pago de deudas y en el proceso de toma de decisiones financieras acertadas, disminución del nivel de actividades operativas, venta forzada de activos necesarios para el proceso productivo; aunado a que se puede producir una disminución en la rentabilidad, el no aprovechamiento de oportunidades de expansión, descontrol en las operaciones, inversiones y hasta puede llevar la empresa a la quiebra.

\subsection{Eficiencia en la actividad empresarial}

En toda actividad empresarial es indispensable conocer la eficiencia con la que se utilizan los insumos, los activos y se gestionan los procesos; por ello es importante destacar que la eficiencia está referida a la relación que existe entre el valor del producto generado y los factores de producción utilizados para obtenerlo.

No obstante, existen indicadores de eficiencia que miden el nivel de ejecución del proceso productivo, centrándose en el cómo se realizan las actividades y en el rendimiento generado por los recursos utilizados.

El análisis financiero permite medir la eficiencia con la cual una organización utiliza sus activos y otros recursos, mediante los denominados indicadores de eficiencia o actividad; los mismos están enfocados básicamente a determinar la celeridad con la que cuentas específicas se transforman en ventas o efectivo; es decir, son valores que muestran que tan efectivamente son manejados los activos totales, activos fijos, inventarios, cuentas por cobrar, el proceso de cobranzas y cuentas por pagar.

Entre los indicadores de eficiencia o actividad se encuentran la rotación de activos totales, rotación de activos fijos, la rotación del inventario, rotación de las cuentas por cobrar, el período promedio de cobro y el período promedio de pago (Figura 2).

La rotación de activos totales indica la capacidad que posee una empresa para la utilización de sus activos totales en la obtención de ingresos; es decir; está referida a la eficiencia en el manejo de activos para generar mayores ventas. Está expresada en el número de veces que una empresa renueva sus activos totales durante un ejercicio económico determinado; mientras más alta es la rotación de 
Análisis financiero: una herramienta clave para una gestión financiera eficiente Nava Rosillón, Marbelis Alejandra

Figura 2

Indicadores de Eficiencia o Actividad

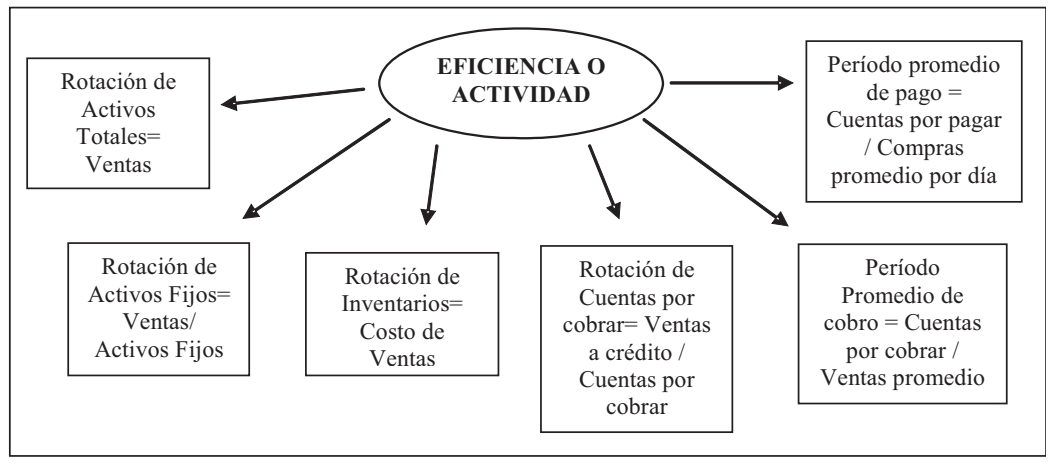

Fuente: Elaboración propia.

activos totales mayor resulta el nivel de eficiencia en el uso que hace la empresa de los bienes y derechos que posee.

De igual manera, la rotación de activos fijos expresa la eficiencia de la empresa para generar ingresos a través de la inversión que realice en activos fijos (edificaciones, instalaciones, maquinarias, equipos). Es un valor que expresa el número de veces que la empresa renueva sus activos fijos en un año; mientras mayor resulte el valor de este indicador, implica una utilización más eficiente de los bienes que posee la organización.

Un aspecto de singular importancia radica en que los inventarios, en cualquier organización, constituyen la cantidad mínima de productos o artículos disponibles que se requiere para satisfacer la demanda de los clientes; razón que denota la relevancia de determinar la rotación de inventarios, la cual mide la liquidez del inventario disponible; es decir, refleja la capacidad de la gerencia de convertir eficientemente el inventario en efectivo o cuentas por cobrar.
Este, es un indicador que expresa el número de veces que se rota el inventario en un año; mientras más alta sea la rotación del inventario significa que en la empresa se gestiona eficientemente y se mantiene constantemente mercancía nueva; contrario a esto, el mantenimiento de bajo inventario se traduce en productos y artículos obsoletos; por ello, la rotación de inventarios permite conocer el manejo del mismo, detectando las ineficiencias que puedan existir en su gestión.

La rotación de las cuentas por cobrar proporciona información acerca de la cantidad de veces que, en promedio, las ventas a crédito son cobradas, durante un período de tiempo específico, convirtiendo su saldo en efectivo. Van Horne (2003), señala que este indicador muestra el número de veces que las cuentas por cobrar han sido transformadas en efectivo reflejando el éxito de la empresa en el reembolso de sus ventas a crédito. Sí este indicador alcanza un valor alto sugiere el establecimiento de políticas de cobro gestionadas en forma eficiente. 
En tanto, el período promedio de cobro se refiere a la cantidad de tiempo promedio en que una empresa recupera sus ventas a crédito; es decir, el número de días en que el efectivo generado de las ventas a crédito permanece en manos de los clientes. El período promedio de cobro, generalmente, es de 30 días, sí este indicador alcanza un valor muy alto o muy bajo es desfavorable para la empresa, ya que puede sugerir ineficiencias en las políticas de crédito y de cobro.

En cuanto al período promedio de pago, Brigham y Houston (2006) plantean que es el tiempo promedio transcurrido entre la compra de materiales, mano de obra y el pago en efectivo de esa compra.

En términos generales, es un indicador de eficiencia que expresa es el tiempo requerido por una empresa para cancelar sus compromisos o compras a crédito; esto se traduce en el número de días que una empresa tarda en pagar sus deudas. Su cálculo es importante para la evaluación de la empresa solicitante de compras a crédito, ya que permite determinar que está en capacidad de pagarlas a tiempo.

\subsection{Capacidad de endeudamiento}

Antes de tomar la medida de hacer uso de financiamiento, el gerente financiero debe revisar la capacidad de pago de la empresa; pues, es la manera de determinar el volumen máximo de endeudamiento en que esta se encuentra.

No obstante, la situación de endeudamiento de una empresa indica el monto de dinero que terceros aportan para generar beneficios en una actividad productiva. Esto se traduce en que el nivel de deuda de una empresa está expresado por el importe de dinero que realicen personas externas a la empresa, llamadas acreedores, cuyo uso está destinado a la obtención de utilidades.

Este nivel de deuda puede ser determinado mediante el análisis financiero, el cual se fundamenta en el cálculo y aplicación de una serie de indicadores o razones financieras que miden la capacidad de una empresa para adquirir financiamiento, estableciendo sí ésta puede ser financiada por sus accionistas o por acreedores; asimismo, evaluar la capacidad que presenta para cumplir con las obligaciones contraídas con terceras personas tanto a corto como a largo plazo.

Entre los indicadores de endeudamiento más destacados se incluyen: razón deuda, razón pasivo circulante y pasivo total, razón pasivo a largo plazo y pasivo total y razón cobertura de intereses (Figura 3).

La razón deuda refleja la proporción de activos totales financiados por los acreedores de la empresa y mientras más alto sea su valor significa que los acreedores han aportado una gran suma de dinero para las operaciones empresariales esperando obtener beneficios.

La razón pasivo circulante y pasivo total, simplemente expresa la proporción de los pasivos totales correspondientes a las deudas o pasivos cuyo vencimiento es menor a un año; en tanto, la razón pasivo a largo plazo y pasivo total, indica la proporción de los pasivos totales contraída por obligaciones a ser canceladas en un lapso de tiempo mayor a un año.

En cuanto a la razón cobertura de intereses, arroja un valor que refleja la capacidad de la empresa para cancelar los 
Análisis financiero: una herramienta clave para una gestión financiera eficiente Nava Rosillón, Marbelis Alejandra

Figura 3

Indicadores de endeudamiento

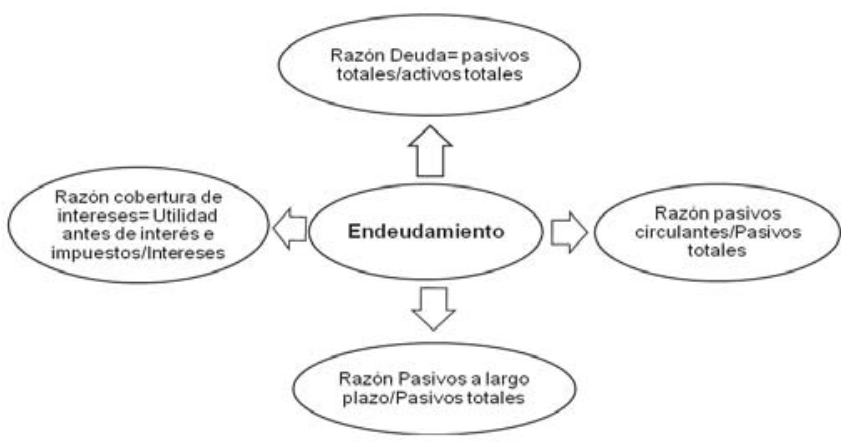

Fuente: Elaboración propia.

intereses del endeudamiento incurrido, mientras más alto sea este valor mayor será la capacidad de la empresa para pagar.

Ahora bien, el gerente financiero presta mayor atención a las deudas de largo plazo, ya que estas significan el compromiso de la empresa para cancelar los intereses en el transcurso del tiempo y el capital en la fecha correspondiente al vencimiento. Por su parte, los accionistas o propietarios enfatizan en su capacidad de reembolsar el monto de la deuda; mientras que los acreedores se interesan por el grado de endeudamiento de la empresa, ya que en la medida que el nivel de deuda sea mayor, la probabilidad de que la empresa no esté en condiciones de cumplir con el pago del capital más los intereses también será mayor.

Sin embargo, utilizar financiamiento tiene sus implicaciones sobre el riesgo y el rendimiento en cualquier empresa; puesto que la inversión de los accionistas será limitada aún cuando mantengan el control sobre su empresa, los acreedores visualizan el capital de los propietarios para disponer de un margen de seguridad, dado que corren con el mayor riesgo dentro de la empresa y por último, el rendimiento sobre el capital de los propietarios se puede incrementar o verse apalancado sí la empresa logra un mejor rendimiento sobre las inversiones financiadas por medio de préstamos en relación al interés pagado sobre los mismos.

\subsection{Rentabilidad}

La rentabilidad constituye el resultado de las acciones gerenciales, decisiones financieras y las políticas implementadas en una organización. Fundamentalmente, la rentabilidad está reflejada en la proporción de utilidad o beneficio que aporta un activo, dada su utilización en el proceso productivo, durante un período de tiempo determinado; aunado a que es un valor porcentual que mide la eficiencia en las operaciones e inversiones que se realizan en las empresas.

A través del análisis financiero se pueden determinar los niveles de rentabilidad de un negocio; pues, permite eva- 
luar la eficiencia de la empresa en la utilización de los activos, el nivel de ventas y la conveniencia de efectuar inversiones, mediante la aplicación de indicadores financieros que muestran los efectos de gestionar en forma efectiva y eficiente los recursos disponibles, arrojando cifras del rendimiento de la actividad productiva y determinando si ésta es rentable o no. Entre estos indicadores se encuentran el rendimiento sobre las ventas, el rendimiento sobre los activos y el rendimiento sobre el capital aportado por los propietarios (Figura 4).

El rendimiento sobre las ventas es un indicador financiero que expresa la utilidad que obtiene la organización en relación con sus ventas e indica el costo de las operaciones y las fluctuaciones que pueda sufrir tanto el precio como el volumen de los productos.

Por su parte, el rendimiento sobre los activos está enfocado en medir la efectividad con que se utilizan los activos necesarios para el proceso de producción; esto se traduce en la proporción de las ganancias obtenidas por la empresa dada su inversión en activos totales (activos circulantes + activos fijos). En tanto, el rendimiento sobre el capital muestra, en términos porcentuales, las ganancias generadas dado el capital aportado por los accionistas o propietarios del negocio.

Así, los indicadores de rentabilidad muestran los retornos netos obtenidos por las ventas y los activos disponibles, midiendo la efectividad del desempeño gerencial llevado a cabo en una empresa.

\section{Análisis financiero y sus técnicas}

El gerente financiero al momento de realizar un análisis financiero en la organización dispone de diferentes técnicas que puede aplicar para analizar e interpretar en profundidad las cifras arrojadas por los indicadores financieros, esto le permite dar respuestas a aquellas preguntas que puedan surgir una vez calculados los mismos.

Figura 4

Indicadores de Rentabilidad

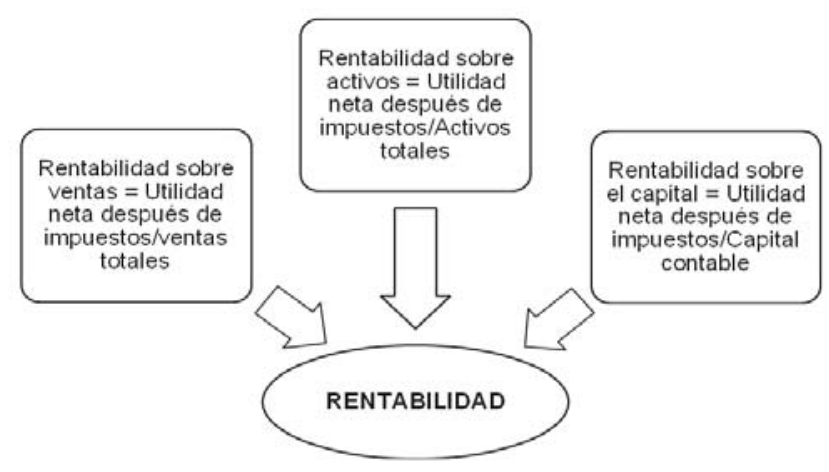

Fuente: Elaboración propia. 
Análisis financiero: una herramienta clave para una gestión financiera eficiente Nava Rosillón, Marbelis Alejandra

Entre las técnicas analíticas más utilizadas se encuentran las siguientes:

\subsection{Análisis comparativo}

Comparar las razones financieras de una empresa permite determinar promedios, tendencias y variaciones ocurridas en el transcurso del tiempo. Según Gitman (2003), existen dos tipos de comparaciones de razones: el análisis seccional y el análisis de series de tiempo que permiten una mejor interpretación del valor de la razón o indicador.

El análisis seccional consiste en comparar los indicadores financieros de diferentes empresas del mismo ramo o con estándares de referencia disponibles acerca del sector productivo al que pertenece la empresa, correspondiente a un ejercicio económico específico. Mediante esta comparación una empresa logra identificar sus fortalezas y debilidades, detectando así aquellas áreas que deben ser mejoradas o fortalecidas.

Este análisis muestra cualquier variación positiva o negativa que pueda presentar algún indicador en relación al promedio del sector, lo cual refleja la existencia de un problema financiero; por ello, la gerencia debe realizar un análisis más profundo para establecer las estrategias más idóneas que les permitan solventar cualquier situación que pueda obstaculizar el proceso productivo.

Por otra parte, el análisis de series de tiempo permite evaluar el desempeño financiero de la empresa en el presente y compararlo con el desempeño en períodos pasados; es decir, compara los indicadores financieros de manera tal, que permite conocer las tendencias de las mismas a través del tiempo; pues, así logra determinar el crecimiento con respecto a lo planificado, las mejoras o deterioros producidos en la situación financiera de la empresa de un año a otro; detectando cualquier variación significativa en las cifras de los indicadores de un ejercicio económico a otro, esto conlleva a identificar los problemas financieros existentes, conocer sus causas y tomar las medidas correctivas más adecuadas para solucionarlos y así lograr mejores resultados en el futuro.

En este orden de ideas, es importante destacar que particularmente, en Venezuela, no se disponen de indicadores financieros de referencia para ningún sector productivo que sirvan como valores estándares, lo cual es contraproducente para la comparación de los indicadores financieros con el promedio del sector al cual pertenece la empresa estudiada; por ello, el análisis comparativo en nuestro país se ve limitado sólo a la aplicación del análisis de series de tiempo.

\subsection{Análisis dupont}

El análisis Dupont constituye una técnica de investigación (Gitman, 2003) encaminada al hallazgo de las tareas administrativas responsables del desempeño financiero de la empresa, tomando en consideración todos los elementos de las actividades financieras del negocio. Tiene como punto de partida la interrelación de ciertos indicadores financieros, cuya interrelación genera el índice que mide la capacidad de la empresa para obtener sus utilidades.

En sí, es un sistema que facilita a la empresa la realización de un análisis inte- 
gral de indicadores financieros específicos, expresando la manera en que estos indicadores interactúan entre sí para determinar el rendimiento sobre los activos; esto sugiere que permite descomponer el rendimiento del capital contable en un elemento de eficiencia en la utilización de activos, en un mecanismo de utilidad sobre las ventas y también de uso de apalancamiento financiero.

El análisis Dupont consiste en combinar el estado de resultados y el balance general, de modo que se obtengan dos medidas globales de rentabilidad: el rendimiento sobre la inversión (ROI) y el rendimiento sobre el capital contable (RSC).

EI ROI se mide a través de las utilidades operativas, es el resultado de multiplicar el margen de utilidad neta con la rotación de activos totales; mientras que el RSC se obtiene de multiplicar el rendi- miento sobre los activos o inversión por el apalancamiento financiero; éste último es el resultado de relacionar los activos totales con el capital contable de la empresa (Figura 5).

En términos generales, el análisis Dupont se fundamenta principalmente en la interacción entre el margen de utilidad sobre las ventas, la rotación de activos y el apalancamiento para determinar el grado de rendimiento sobre el capital contable.

De este modo, el análisis Dupont constituye una herramienta altamente relevante para la planificación financiera empresarial; puesto que proporciona toda la información pertinente referente a la rentabilidad generada sobre la inversión realizada y sobre el capital contable aportado para ello; a partir de lo cual permite realizar planes financieros tanto a

Figura 5

\section{Sistema Dupont}

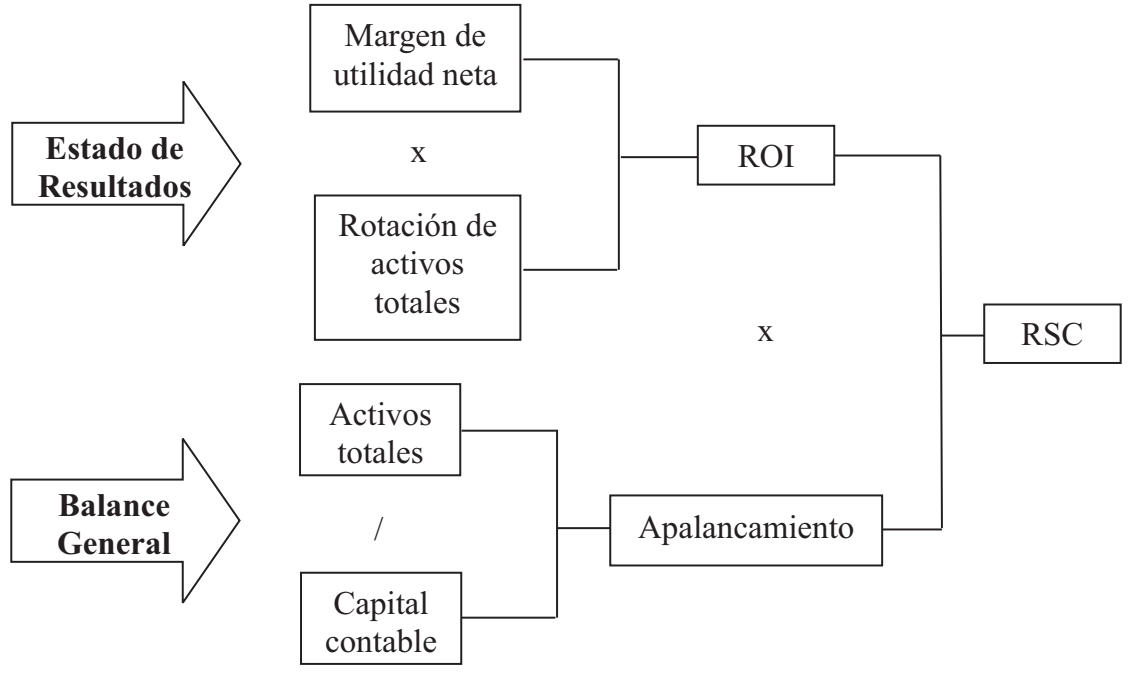

Fuente: Elaboración propia. 
Análisis financiero: una herramienta clave para una gestión financiera eficiente Nava Rosillón, Marbelis Alejandra

largo como a corto plazo acordes con la condición financiera actual de la empresa, llevando a una toma de decisiones financieras más adecuada para alcanzar un mejor desempeño en todas las áreas de la organización.

\subsection{Análisis discriminante}

El análisis discriminante constituye una técnica estadística con uso predominante para analizar la información financiera en la década de los años 80, fue aplicada por Edward Altman en 1968 para la selección y evaluación de aquellos indicadores financieros que permiten distinguir entre empresas financieramente exitosas y empresas con rumbo a la quiebra.

Básicamente, este análisis consiste en clasificar observaciones previamente obtenidas, cuya clasificación tiene como punto de partida un conjunto de variables que caracterizan los individuos $u$ objetos que se pretenden estudiar (Elizondo y Altman, 2003). Desde el punto de vista financiero, esta técnica se fundamenta en la combinación y estudio de los indicadores o razones financieras de una empresa o de un grupo de empresas.

Inicialmente, Altman desarrolló un modelo para predecir la quiebra de las empresas y fue llamado $Z$ de Altman, que actualmente representa el resultado de aplicar el análisis discriminante a un conjunto de indicadores financieros cuyo propósito es clasificar las empresas en dos grupos: bancarrota y no bancarrota (Elizondo y Altman, 2003); aún cuando este Economista también ha desarrollado modelos para la evaluación de créditos.
El enfoque del análisis discriminante muestra la correlación entre indicadores individuales, escogiendo aquellos que contribuyen más al valor discriminante denominado valor de $Z$, cuyo enfoque ha sido aceptado considerablemente.

La función discriminante que resultó del valor $Z$ de Altman es la siguiente: $Z=6.56 \times 1+3.26 \times 2+6.72 X 3+1.05 X 4$; donde:

$X=$ Índices seleccionados para conformar el valor Z (Cuadro 1).

\section{Cuadro 1} Función discriminante

\begin{tabular}{cc}
\hline $\begin{array}{c}\text { Valores } \\
\text { X }\end{array}$ & Relación \\
\hline X1 & Capital de Trabajo / Activo Total \\
X2 & Utilidades Retenidas / Activo Total \\
X3 & Utilidad antes de impuestos / \\
& Activo Total \\
X4 & Total patrimonio / Pasivo Total \\
\hline
\end{tabular}

Por su parte, los límites de referencia son los siguientes:

$Z>=2.60$ Baja probabilidad de quiebra; puede tratarse de una empresa financieramente bien gerenciada que no muestra posibilidad de llegar a la quiebra en un futuro cercano; es decir, implica que es una empresa saludable desde la perspectiva financiera.

$Z<=1.10$ Alta probabilidad de quiebra.

$1.10<Z<2.60$ Zona gris o de incertidumbre; donde puede ocurrir que las empresas con valores $Z$ ubicados entre estos límites, sean muy buenas pero se encuentre gerenciadas en forma ineficiente, o empresas malas pero gerenciadas con la mayor eficiencia. 
Estos parámetros indican que mientras mayor sea el valor de Z, menor es la probabilidad de que la empresa se vaya a la quiebra.

Resulta importante destacar que este modelo puede ser aplicado para un grupo de empresas o para una empresa individual. Para un grupo de empresas, como un primer paso se debe calcular cada uno de los indicadores que contiene el modelo para cada empresa de la muestra estudiada; posteriormente, se calcula el valor promedio de cada índice en el grupo de empresas; y por último, se sustituye en la función discriminante para obtener el valor de $Z$.

Cuando se trata de aplicar el modelo en una sola empresa, se procede de igual forma, se calcula cada indicador del modelo y el resultado se sustituye en la función discriminante para obtener el valor de Z. Luego, se comparan los valores de $Z$ obtenidos con los parámetros de referencia de manera de diagnosticar la situación financiera de la empresa o empresas estudiadas.

\section{Análisis financiero e inflación}

La actividad empresarial de cualquier país está expuesta a factores económicos que influyen en su desarrollo y por ende en sus resultados, como es el caso específico de la inflación. Este fenómeno es una realidad económica que persiste en Venezuela desde hace años y afecta los precios, costos, sueldos y salarios, valor de las propiedades y todo lo relacionado con el dinero.

Mochón (2001) define la inflación como el crecimiento generalizado y conti- nuo de los precios de los bienes y servicios generados en una economía.

En base a esta definición, los estados financieros de una empresa deben reconocer los efectos del fenómeno inflacionario; puesto que registran las operaciones en el valor monetario que tienen en el momento en el cual se adquieren los bienes y servicios; esto genera como consecuencia, que en economías inflacionarias con el transcurso del tiempo las operaciones queden expresadas en valores históricos; por ello, se hace necesario reexpresar los estados financieros para actualizar las cifras de las operaciones con el valor correspondiente a la fecha de cierre del último ejercicio económico.

Para Redondo (1993) reexpresar los estados financieros significa transformar sus valores históricos de diferentes poderes adquisitivos en unidades monetarias equivalentes con el mismo poder adquisitivo a la fecha de actualización. En otras palabras, el denominado ajuste por inflación consiste en una corrección monetaria que permite expresar los estados financieros realizados inicialmente en una moneda inestable por los efectos de la inflación, en otra moneda más estable, permitiendo conocer la utilidad real de las operaciones de la empresa, comparando los patrimonios inicial y final expresados en las mismas unidades monetarias.

En Venezuela, para reexpresar los estados financieros se emplean el Método de Nivel General de Precios o Precios Constantes y el Método Mixto. El primero es ampliamente conocido y aceptado en el mundo y se fundamenta en actualizar los estados financieros a una determinada fecha tomando como base el nivel general de precios; homogeneizando el po- 
Análisis financiero: una herramienta clave para una gestión financiera eficiente Nava Rosillón, Marbelis Alejandra

der adquisitivo de la moneda en la fecha de presentación de la información financiera (Redondo, 1993).

Según la Declaración de Principios Contables No 10 (DPC-10), el segundo método consiste en la aplicación integral de un índice general de precios a todas las partidas de los estados financieros (Método del Nivel General de Precios) y luego reemplazar los valores corrientes aplicables (Método de Costos Corrientes). Este ha sido adaptado al caso venezolano cuando en el año 1991, la mayoría de las empresas revaluaron sus activos fijos para proteger su patrimonio debido al alto nivel de inflación.

Ajustar los estados financieros por inflación tiene mucha utilidad para una organización, ya que suministran información más relevante que los estados financieros históricos; pues, permite realizar una revaluación de los activos fijos empresariales como una forma de actualizar el valor patrimonial reconociendo este último con un valor más cercano a la realidad mediante valores más actualizados y reales de los activos fijos, inventarios e inversiones; siendo estos los activos más relevantes de cualquier empresa.

Del mismo modo, al ajustar por inflación el resto de las partidas de los estados financieros y calcular los indicadores financieros se obtienen cifras más actualizadas, válidas y reales que muestren la verdadera situación financiera de la organización; ya que en economías inflacionarias como la economía venezolana los estados financieros basados en costos históricos no permiten que la información sea veraz, actual, relevante y precisa (De la Hoz et al., 2008); ya que cuando los precios aumentan en forma general, los valores se distorsionan.

Por lo tanto, la situación patrimonial de cualquier empresa que opere en entornos inflacionarios sufre ineludiblemente los efectos de este fenómeno; de allí, la importancia de la reexpresión de estados financieros para el análisis financiero; ya que realizar el análisis financiero con estados financieros expresados en cifras históricas origina variaciones de incrementos o disminuciones en términos absolutos y relativos irreales y se obtendrían resultados diferentes en los indicadores financieros.

Contrario a ello, un análisis financiero basado en cifras actualizadas o reexpresadas arrojará resultados mayormente ajustados a las verdaderas condiciones económicas del entorno, lo que permite determinar la situación financiera actual, visualizar el futuro de la empresa y tomar las decisiones más idóneas para el éxito de la misma.

\section{Análisis Financiero y Normas Internacionales de Información Financiera}

La convergencia contable internacional ha llevado a una toma de conciencia a nivel internacional que ha concebido la aceptación de un proceso de generación de normas y recomendaciones, cuya adopción no es un hecho aislado sino que corresponde a una estrategia relacionada con la globalización e internacionalización de las actividades empresariales, el proceso de armonización y homogeneización internacional de normas y la necesidad de garantizar la transparencia; la 
cual, a su vez, garantiza la confianza en los mercados (Sánchez, 2006).

Razones que llevan al surgimiento de nuevas tendencias encaminadas a establecer normas congruentes con las necesidades de información contable y financiera en el entorno empresarial mundial; normas que constituyen un consenso entre principios europeos y americanos cuyo propósito se centra en facilitar las relaciones financieras y el comercio internacional; pues, se trata de las Normas Internacionales de Contabilidad (NIC) y las Normas Internacionales de Información Financiera (NIIF), las cuales han sufrido una reforma recientemente y que nuestro país se ha pronunciado a adoptar a través de la Federación de Colegios de Contadores Públicos, cuya institución decidió que estas normas serán implementadas por las grandes empresas desde el año 2008, mientras que las pequeñas y medianas empresas (PYMES) se regirán por estas normas a partir del año 2009.

Según Sánchez (2006) explica, estas normas generan cambios en la información financiera registrada en los estados financieros; pues, las NIC y NIIF introducen un marco conceptual coherente con las necesidades de información de los mercados, incrementan la información que debe aparecer en los estados financieros e incluyen nuevas formas de presentación y contenido del balance y del estado de resultados; así como también establecen la elaboración obligatoria de estados contables como el estado de variaciones en el patrimonio neto y el estado de flujo de efectivo o de tesorería; y la elaboración voluntaria del estado de información segmentada, estado de ga- nancia por acción, estado de cambios en estimaciones y errores, entre otros.

Dado lo anteriormente planteado, es necesario señalar que en este artículo, la idea de relacionar las NIIF con el análisis financiero no es detallar la reforma de estas normas, sino precisar que la adopción de la misma genera efectos al momento de aplicar el análisis financiero en la actividad empresarial. Pues, esta reforma introduce una nueva terminología en la presentación de los estados financieros, especialmente para el balance general, lo cual no indica que cambie el significado de los conceptos, sino que se utiliza un nuevo lenguaje; por ello, se debe prestar gran atención para evitar confusiones en la aplicación y cálculo de los indicadores financieros requeridos para evaluar la situación económica y financiera de las empresas.

Por ello, en Venezuela, todo gerente financiero debe estar a la vanguardia de la reforma de las NIIF y NIC, debe conocer en profundidad los nuevos planteamientos; ya que se tratan de normas más complejas que generarán variaciones muy marcadas tanto en el capital propio como en el estado de resultados, lo cual repercute en los indicadores financieros utilizados, induciendo diferencias en los enfoques y criterios existentes habitualmente. Cabe considerar que la implementación de estas normas presenta sus beneficios tanto a nivel mundial como a nivel empresarial; puesto que buscan mejorar el ambiente económico de manera que sea más atractivo para los inversionistas, crear las condiciones más adecuadas para que las empresas puedan crecer y expandirse, tratan de armonizar la información para que exista comparabilidad, 
Análisis financiero: una herramienta clave para una gestión financiera eficiente Nava Rosillón, Marbelis Alejandra

transparencia, uniformidad, eficiencia, competitividad y una mayor confianza en el contexto empresarial.

\section{Conclusiones}

El estudio concerniente al análisis financiero como herramienta clave para una gestión financiera eficiente ha permitido indagar importantes aspectos de esta técnica gerencial, incluyendo, una breve referencia acerca de la repercusión de la implementación de la nueva reforma de las NIIF en su aplicación.

Los autores referidos en este artículo coinciden en muchos aspectos acerca del análisis financiero; pues, algunos de ellos consideran que es fundamental para evaluar la situación actual de la empresa y predecir su desempeño futuro; generalmente lo denominan análisis de los estados financieros; y la mayoría alega que se basa en la aplicación y cálculo de indicadores financieros que permiten conocer la situación en lo que respecta a liquidez, eficiencia, endeudamiento, rendimiento y rentabilidad.

Aunado a esto, los autores aportan nociones que permiten identificar aquellos aspectos que caracterizan el comportamiento gerencial, económico y financiero de una empresa, donde el análisis financiero representa el medio más idóneo para interpretar y evaluar la información contable que refleja el manejo de los recursos financieros disponibles para el proceso productivo, lo cual lleva implícito la detección de las deficiencias y desviaciones ocurridas durante la gestión empresarial.

El análisis financiero es un tema muy extenso y, sin lugar a dudas, consti- tuye una herramienta fundamental para que en una organización se logre una gestión financiera eficiente. Realizarlo es de vital importancia para el desempeño financiero de la empresa; pues, se trata de una fase gerencial analítica basada en información cualitativa y cuantitativa cuya aplicación permite conocer las condiciones de la salud financiera de la organización mediante el cálculo de indicadores, que tomando como fundamento la información registrada en los estados financieros, arrojan cifras que expresan el nivel de liquidez, el grado de solvencia, la eficiencia en el manejo de los activos e inversiones, la capacidad de endeudamiento, así como también, el nivel de rentabilidad y rendimiento obtenido sobre las ventas, activos, inversiones y capital.

Además, el análisis financiero dispone de técnicas cuya aplicación permite un análisis e interpretación más profunda, que va desde determinar la gestión de los recursos financieros disponibles en el presente hasta la predicción de la quiebra de la empresa en el futuro más próximo.

No obstante, las evidencias empíricas reflejan que mediante el análisis financiero, las empresas detectan problemas en el proceso de cobranza de sus ventas a crédito, en el pago de sus compras a crédito, en el uso que realizan de sus maquinarias, equipos y construcciones, así como de su efectivo disponible, inventario, ventas, gastos y ganancia, determinando sí su actividad es rentable o no. Del mismo modo, el análisis financiero permite determinar sí los planes de acción se están cumpliendo a cabalidad de manera que los objetivos previamente establecidos sean alcanzados eficientemente. 
Por ello, se debe tomar en consideración que cuando en una economía los precios de los bienes y servicios aumentan como consecuencia de la inflación, los valores se distorsionan y por ende las cifras de los estados financieros de las empresas; razón por la cual estos deben ser ajustados para determinar sí la inflación ha afectado los resultados de sus operaciones, ya que el valor de los activos y pasivos varía y los estados financieros expresan información distorsionada; entonces, mediante la reexpresión, los estados financieros proporcionan información válida, actualizada, precisa y adecuada para determinar la situación financiera real de la actividad empresarial y lograr una toma de decisiones más acertadas.

En definitiva, se deduce que el análisis financiero es una herramienta trascendental para determinar la situación financiera de una organización, de modo que se logre una gestión financiera eficiente; para ello resulta imprescindible llevar un control adecuado del uso de los activos y de los recursos financieros que están destinados para las inversiones, por lo cual se debe realizar un análisis minucioso de cada uso dado a los fondos disponibles, se trate de recursos propios o provenientes de terceros.

Para tal fin, se requiere una persona capacitada que efectúe el análisis financiero, que posea conocimientos de alto nivel en el área financiera para aplicar técnicas y procedimientos analíticos adecuados que permitan obtener los mejores resultados y alcanzar el objetivo de evaluar el entorno económico y financiero de la empresa, detectar cualquier obstáculo existente, tomar las mejores decisio- nes e implementar acciones correctivas, que a su vez permitan alcanzar un desempeño financiero efectivo que lleve a la empresa al éxito.

A partir de este estudio, se considera necesario que los empresarios venezolanos deben estar dispuestos a asumir los cambios existentes en el entorno financiero, estar a la vanguardia de todas las herramientas gerenciales que permitan analizar ampliamente la actividad que realizan, para conocer en qué condiciones está operando su empresa y como deben enfrentar el futuro, y así lograr una gestión financiera eficiente, lo cual requiere la aplicación del análisis financiero, ya que se trata de un proceso de reflexión que lleva a evaluar cualitativa y cuantitativamente desde el punto de vista financiero la empresa en el presente y en el pasado, determinar los resultados de sus operaciones y estimar su situación y su actuación en el futuro.

\section{Referencias Bibliográficas}

Brigham, Eugene y Houston, Joel (2006). Fundamentos de Administración Financiera $\left(10^{\mathrm{a}}\right.$ ed.). México. Cengage Learning Editores. 831 pp.

De la Hoz, Betty; Uzcátegui, Sigilgredo; Borges, Jesús y Velazco, Angel (2008). La Inflación como Factor Distorsionante de la Información Financiera. Revista Venezolana de Gerencia. Año 13. No 44. Venezuela. Centro de la Empresa. Universidad del Zulia. $556-572$ pp.

Elizondo, Alan y Altman, Edward (2003). Medición Integral del Riesgo de Crédito. Limusa. 200 pp.

Federación de Colegios de Contadores Públicos (2000). Declaración de Princi- 
Análisis financiero: una herramienta clave para una gestión financiera eficiente Nava Rosillón, Marbelis Alejandra

pios de Contabilidad Número 10. (DPC-10). Caracas. Venezuela.

Gitman, Lawrence (2003). Principios de Administración Financiera. $\left(10^{\mathrm{a}} \mathrm{ed}\right.$.) México. Prentice Hall. 631 pp.

Hernández, José Luis (2005). Análisis Financiero. Perú. Disponible en: www.gestiopolis.com/canales5/fin/anfinancier.htm. Consulta: 07 de Septiembre de 2008.

Hurtado; Jaqueline (2007). El Proyecto de Investigación. Metodología de la Investigación Holística. ( $5^{\mathrm{a}}$ ed.). Caracas, Venezuela. Quirón. 183 pp.

Mallo, Carlos y Merlo, José (1995). Control de Gestión y Control Presupuestario. España. Mc Graw Hill. 414 pp.

Mochón, Francisco. (2001). Economía. Teoría y Política. (4 ${ }^{a}$ ed.). España. Mc Graw Hill.
Pacheco, Juan, Castañeda, Widberto y Caicedo, Carlos (2002). Indicadores Integrales de Gestión. Colombia. Mc Graw Hill. 184 pp.

Redondo, Angel (1993). Curso Práctico de Contabilidad General y Superior. ( $3^{\mathrm{a}}$ ed.). Tomo II. Venezuela.

Rubio D., Pedro (2007). Manual de Análisis Financiero. España. Universidad de Málaga. Edición electrónica. Disponible en: http:/www.eumed.net/libros/ 2007a/255. Consulta: 31 de Julio de 2008.

Sánchez, José Luis (2006). Importancia de las NIC/NIIF para el Análisis Financiero. Disponible en: www.iica.cl/ SAC/Descargas/Consecuencias. 30 de Julio de 2008.

Van Horne, James y Wachowicz, John (2003). Fundamentos de Administración Financiera $\left(11^{\mathrm{a}}\right.$ ed.). México. Prentice Hall. 743 pp. 